\title{
Globe
}

Revue internationale d'études québécoises

\section{Bernard Andrès et Gérard Bouchard (dir.) : Mythes et sociétés des Amériques, Montréal, Québec Amérique, 2007}

\section{Jean-François Chassay}

Volume 12, numéro 1, 2009

URI : https://id.erudit.org/iderudit/1000776ar

DOI : https://doi.org/10.7202/1000776ar

Aller au sommaire du numéro

Éditeur(s)

Globe, Revue internationale d'études québécoises

ISSN

1481-5869 (imprimé)

1923-8231 (numérique)

Découvrir la revue

Citer ce compte rendu

Chassay, J.-F. (2009). Compte rendu de [Bernard Andrès et Gérard Bouchard (dir.) : Mythes et sociétés des Amériques, Montréal, Québec Amérique, 2007]. Globe, 12(1), 176-179. https://doi.org/10.7202/1000776ar d'utilisation que vous pouvez consulter en ligne.

https://apropos.erudit.org/fr/usagers/politique-dutilisation/ 
du rapport semble avoir trouvé l'oreille du Législateur, ce n'est pas une raison pour considérer comme inutile ou inopérant l'ensemble de ce rapport. J'ai eu le privilège de participer aux travaux de la Commission, j'y ai trouvé un laboratoire de philosophie sociale et politique d'une exceptionnelle fécondité et je pense que, une année plus tard, il est temps de retourner aux analyses de ce rapport et d'en discuter soigneusement les conclusions.

Georges Leroux

Université du Québec à Montréal

\section{Bernard Andrès et Gérard Bouchard (dir.) Mythes et sociétés des Amériques \\ Montréal, Québec Amérique, 2007.}

L'intérêt que nous portons, au Québec, à l'Amérique (et en particulier aux Etats-Unis) n'est pas neuf. En se limitant au plan culturel, selon une définition assez stricte du terme, il est certainement possible de remonter au moins jusqu'à l'Institut canadien. La réflexion sur les Amériques, sur ce qu'on nomme maintenant tout naturellement "l'américanité ", est évidemment beaucoup plus récente, datant d'à peine quelques décennies. En gardant en tête le fait que les dates précises, lorsqu'il s'agit de parler du développement d'un concept, ont toujours quelque chose d'un peu aléatoire, on peut se demander néanmoins si la défaite du " oui » en mai 1980 n'a pas un rôle symbolique à jouer dans ce renouvellement d'intérêt pour les Amériques, tant il est vrai que le référendum était associé au "fait français " et, par métonymie, à la France elle-même - par conséquent à l'Europe. Se tourner vers les "terres intérieures" (Pierre Nepveu a intitulé un de ses essais Intérieurs du Nouveau Monde) plutôt que de l'autre côté de l'Atlantique aurait été une façon d'oublier ce qui avait été ressenti comme une défaite par une nette majorité d'écrivains et d'intellectuels francophones. Sans aller trop loin dans cette intuition, il faut rappeler que les romans "américains" publiés par des francophones québécois dans les années subséquentes se sont multipliés. On peut même dire que Jacques Poulin, auteur jusque-là relativement peu connu, a été vraiment consacré après la parution en 1984 de Volkswagen Blues, road book se déroulant de Gaspé à San Francisco, devenu un peu le roman phare de la décennie.

Une trentaine d'années après ce nouveau souffle donné aux analyses de nos relations intellectuelles avec les Amériques, Mythes et societés 
des Amériques, collectif dirigé par Gérard Bouchard et Bernard Andrès, euxmêmes deux spécialistes bien connus de la question, apparaît comme une somme, mise au point ou mise à jour de la question.

Le propos sur l'imaginaire des Amériques se cristallise dans cet ouvrage sur la place du mythe et sur sa persistance encore aujourd'hui. Malgré cette volonté de canaliser la réflexion, les risques de dispersion étaient grands en abordant un sujet aussi vaste. Comme Nova Doyon l'écrit dans son texte : "Le mythe se présente souvent sous une forme hétéroclite, polyvalente et polysémique" (p. 242). Il est sans doute exagéré de dire que les définitions peuvent varier à l'infini, mais elles peuvent être suffisamment dissemblables pour rendre infructueuses les convergences espérées.

Je me permettrai d'en donner ici une qui ne se retrouve pas dans l'ouvrage. Elle a été proposée par Claude Lévi-Strauss : « l'objet du mythe est de fournir un modèle logique pour résoudre une contradiction (tâche irréalisable quand la contradiction est réelle) $)^{8}$. "On pourrait l'interpréter en proposant que le mythe rend manifeste ce qui ne peut se résoudre, une dialectique impossible. Il fournit une explication acceptable, pour un groupe donné, à ce qui par ailleurs semble échapper à tout ordre de causalité.

Dans leur excellente présentation, Bernard Andrès et Gérard Bouchard synthétisent les tendances qui dominent à la lecture des 12 chapitres qui composent le livre, au point où le commentateur, un peu dépité, a l'impression que le travail a été fait pour lui ! Ils dégagent notamment quelques interrogations qui apparaissent primordiales : la façon dont naît et s'élabore le mythe américain, les dérives du mythe, le topos de l'échec, la capacité du mythe à transcender les contradictions. Il me semble que c'est cette dernière (et voilà pourquoi je tenais à citer Lévi-Strauss) qui paraît la plus importante. Car, en effet, l'entrelacement des éléments contradictoires qui composent le substrat de l'Amérique s'impose de manière souvent spectaculaire dans les textes et seul le mythe semble en mesure de dénouer ces contradictions en les transcendant. Chaque époque fabrique ses propres mythes et ceux-ci répondent à des besoins précis. Mais il s'agit souvent d'adaptations de phénomènes anciens que l'Amérique ne peut que réitérer. Maximilien Laroche en fait une éloquente démonstration dans son texte où il note, au début de sa conclusion : "Un mythe [...] ne fait pas que s'adapter à un lieu. En se diffusant, c'est-à-dire en étant connu ailleurs, il se modifie au fil du temps et, revenant ainsi modifié à son point de départ, il ne peut

$$
4+4
$$

8. Claude LÉVI-STRAuS, Anthropologie structurale, Paris, Plon, 1958, p. 254. 
manquer de transformer sa version originale " (p. 128). L'Amérique vit, encore aujourd'hui, de contradictions qui forment parfois une véritable double contrainte dont, à un certain niveau d'entendement, seul l'imaginaire peut rendre compte adéquatement, en s'attaquant aux mythes. Prenons en ce sens le premier chapitre, rédigé par Zilá Bernd. Il trouve tout à fait sa place au début de l'ouvrage, puisqu'il concerne la naissance du mythe américain. Mais la figure du nouveau-né croise sans cesse dans la fiction celle de Médée, modèle mythique par excellence de l'infanticide. Rien de surprenant à cela : ce Nouveau Monde que découvrent les Européns est indissociable du meurtre, celui perpétré sur des populations autochtones dont on a longtemps nié non seulement le droit de "propriété ", mais même la valeur au sein de l'espèce humaine. Ce n'est pas pour rien qu'aux États-Unis l'œuvre de Fenimore Cooper joue souvent un rôle fondateur et que l'auteur a toujours eu un rapport extrêmement conflictuel avec son pays (et inversement) : ses livres impliquent la présence de l'autochtone et montrent à quel point le mythe (européen) de l'Amérique se construit sur un rituel sanguinaire. Pas de conquête sans meurtre. Chaque chapitre du collectif, à sa façon, me semble$t$-il, reproduit à partir d'une perspective singulière cette tension propre à l'Amérique que le mythe tente de résoudre.

Il serait injuste de s'arrêter sur un texte de cet ensemble plutôt que sur un autre et un survol très rapide de chacun d'entre ne leur rendrait pas justice. On peut néanmoins mentionner que la dynamique de l'ouvrage permet de toucher aussi bien à des questions assez générales (le pionnier chez Louise Vigeant, "l'invention de la mémoire longue dans les nations du Nouveau Monde " chez Gérard Bouchard), des personnages particuliers ("D'Iberville et le mythe d'une Amérique française " de Bernard Andrès) ou encore des sujets singuliers, pour ne pas dire surprenants, par exemple, le baseball chez Michel Nareau ("Le mythe états-uniens du baseball et ses contradictions dans les Amériques") ou encore le chien dans le texte de Janusz Przychodzen, qui souligne l'étonnement des Européens, à leur arrivée, devant le silence des canidés américains. Texte fort intéressant, dont je regrette seulement (c'est lié au souvenir d'une lecture lumineuse) l'absence du corpus de Dormir au soleil d'Adolfo Bioy Casares?.

Au bout du compte, ce livre est une somme d'abord parce qu'il ne s'agit pas de réactions ponctuelles à un événement. Le résultat ne ressemble pas, par exemple, à des actes de colloque. On a plutôt des textes longs,

$$
+4
$$

9. Adolfo BIOY CASARES, Dormir au soleil, Paris, Gallimard, 1992.

RECENSIONS 
rédigés par des spécialistes qui ont souvent eu l'occasion d'échanger autour de la "question américaine ".

La pensée rationnelle semble être par nature exclusive et rejeter tout ce qui n'en relève pas au rayon des religions et des mythes. Alors même que la vérité scientifique n'est pas toute la vérité sur le monde et que c'est justement le rôle des mythes de dire quelque chose qui ne pourrait être dit autrement ${ }^{10}$.

Cela ne signifie pas qu'on ne peut rétablir la vérité face à une légende ; cela veut dire qu'elle ne suffit ni à sa disparition ni à son obsolescence. Ce livre le rappelle et en fait l'éclatante démonstration.

Jean-François Chassay

Université du Québec à Montréal

\section{François Crépeau et Jean-Philippe Thérien (dir.)}

Penser l'international. Perspectives et contributions des sciences sociales

Montréal, Les Presses de l'Université de Montréal, 2007.

Sous l'égide du Centre d'études et de recherches internationales de l'Université de Montréal, des chercheurs ont été invités à dégager la spécificité de chacune de leurs disciplines dans l'approche de l'international. En dépit ou à cause du cahier des charges soumis aux intervenants, rendre compte de cet ouvrage collectif n'est pas aisé parce qu'il regroupe des éléments hétérogènes de définition de l'international, prend des exemples issus de terrains d'observation ou de réglementations variés et procède à des inventaires lapidaires.

L'introduction part du constat que "l'internationalisation influe sur tous les aspects de la vie universitaire" (p. 7). L'international s'infiltre dans la plupart des disciplines et ne se cantonne plus à la science politique, au droit et à l'histoire. Les auteurs insistent sur les redécoupages et réorientations qui en découlent, sur l'effacement de certaines frontières disciplinaires et nationales. Mais ils considèrent que les " relations diplomatiques et militaires entre États " demeurent parmi les facteurs déterminants (p. 7-8).

$$
+4
$$

10. Sven ORTOLI et Nicolas WITKOWSKI, La baignoire d'Archimede. Petite mythologie de la science, Paris, Seuil, 1996, p. 150. 